\title{
Reduction of the Entrainment Velocity by Cloud Droplet Sedimentation in Stratocumulus
}

\author{
Alberto de Lozar and Juan Pedro Mellado \\ Max Plank Institute for Meteorology, Hamburg, Germany
}

(Manuscript received 1 July 2016, in final form 17 October 2016)

\begin{abstract}
The effect of sedimentation on stratocumulus entrainment is investigated using direct numerical simulations of a cloud-top mixing layer driven by radiative and evaporative cooling. The simulations focus on the meter and submeter scales that are expected to be relevant for entrainment, and the finest grid spacing is $\Delta x=26 \mathrm{~cm}$. The entrainment velocity is investigated from the analysis of the integrated-buoyancy evolution equation, which is exactly derived from the flow evolution equations. The analysis shows that sedimentation interacts with entrainment through two different mechanisms. As previously reported, sedimentation prevents droplets from evaporating in the entrainment zone, which in turn reduces the entrainment velocity. Here it is shown that sedimentation also promotes a positive buoyancy flux that directly opposes entrainment. The strengths of both mechanisms are characterized by two different settling numbers, which allow for predicting which meteorological conditions favor the reduction of entrainment by sedimentation. These new insights allow for including sedimentation in a parameterization of the entrainment velocity. The reduction of the entrainment velocity by sedimentation predicted by the parameterization and observed in the simulations is 3 times larger than previously reported in large-eddy simulations, which implies that meter- and submeterscale turbulence plays an important role in the interaction of entrainment with sedimentation. On the whole, analysis and simulations indicate that stratocumulus entrainment is more sensitive to the cloud droplet number density due to sedimentation than previously thought.
\end{abstract}

\section{Introduction}

Stratocumulus clouds extend for several hundreds of kilometers and have a large albedo and are thus key for Earth's radiation budget (e.g., Wood 2012). Aerosol pollution can significantly change stratocumulus albedo and cloud dynamics, and therefore understanding these changes is important to properly quantify the anthropogenic influence on climate. While the instantaneous effect of increasing aerosols on the albedo is well understood (Twomey 1974), the influence on the stratocumulus dynamics remains more elusive to quantify.

Large-eddy simulations (LES) show that polluted stratocumuli are thicker than unpolluted ones in heavily drizzling conditions but that the opposite tendency prevails for light drizzle and nonprecipitating clouds (Ackerman et al. 2004). These tendencies are explained by comparing two competing mechanisms. On the one hand, aerosol pollution weakens drizzle, which thickens the

Corresponding author e-mail: Alberto de Lozar, adelozar@ gmail.com clouds (Albrecht 1989). On the other hand, aerosol pollution strengthens the entrainment of dry air at cloud top, which thins the cloud. Heavily drizzling clouds are more sensitive to the first mechanism, explaining the different trends. Subsequent LES have confirmed these conclusions ( $\mathrm{Lu}$ and Seinfeld 2005; Sandu et al. 2008; Hill et al. 2008, 2009; Caldwell and Bretherton 2009), proving that this picture is generic for many meteorological conditions, although a systematic quantification is still missing.

The enhancement of stratocumulus entrainment by aerosols pollution is mostly attributed to a lower sedimentation velocity in polluted cases (Ackerman et al. 2004). Sedimentation is expected to weaken entrainment for clean conditions (cloud droplet number density $N_{d} \sim 100 \mathrm{~cm}^{-3}$ ), where the droplet radius $(R \sim 10 \mu \mathrm{m})$ is such that typical sedimentation velocities $\left(u_{s} \sim 1 \mathrm{~cm} \mathrm{~s}^{-1}\right)$ are comparable to the entrainment velocities $\left(w_{e} \sim 1 \mathrm{~cm} \mathrm{~s}^{-1}\right)$. The reduction of entrainment by sedimentation has been studied in LES, where this effect can be isolated. Bretherton et al. (2007) and Ackerman et al. (2009) found that sedimentation alone reduces the entrainment velocity by $3.5 \%$ and $15 \%$ in LES based on the research flights RF01 
and RF02 from the DYCOMS II campaign when assuming a narrow droplet size distribution (DSD) and that the reduction of the entrainment velocity is twice as much for broader DSDs. Bretherton et al. (2007) explained this reduction, arguing that sedimentation removes liquid water from the entrainment zone and therefore weakens the evaporative cooling entrainment amplification. Similarly, Hill et al. (2009) observed a 7\% reduction of the entrainment velocity in LES with bin microphysics (which do not require any assumption about the DSD) when comparing clean and polluted cases, although they attributed part of the reduction to the slower evaporation rate in clean conditions.

The above-cited studies show that sedimentation has the potential for considerably reducing the entrainment velocity, but they also motivate the next questions:

- Can we understand the interplay between entrainment and sedimentation from the integral analysis of the buoyancy evolution equation? Although entrainment velocity parameterizations are often based on the integral analysis of the buoyancy evolution equation (Stevens 2002), to our knowledge sedimentation has never been accounted for in this kind of analysis.

- How sensitive is the effect of sedimentation on entrainment to the meteorological conditions? The sedimentation-entrainment interplay is complex and probably depends on the thermodynamical properties of the stratocumulus top, in addition to the aerosol concentration. It would be useful to identify some nondimensional numbers that indicate how sensitive the entrainment velocity is to changes of aerosol concentrations and thermodynamical properties through sedimentation.

- Are meter and submeter scales relevant for the entrainment-sedimentation interplay? Past studies (Stevens et al. 2005) indicate that stratocumulus entrainment is not fully resolved at current LES resolutions $(\Delta z=5 \mathrm{~m}, \Delta x \geq 35 \mathrm{~m})$, which might have consequences for evaluating the sedimentationentrainment interplay. It is thus desirable to compare LES results with simulations in which the meter and submeter scales are resolved.

In this paper we address these questions by means of direct numerical simulations (DNS) of a cloud-top mixing layer driven by radiative and evaporative cooling, which mimics the stratocumulus top. DNS has been proven a useful tool to study stratocumulus entrainment, because it allows for resolving most length scales that are relevant for entrainment, while at the same time it maintains a large separation of scales between entrainment and boundary layer dynamics (Mellado et al. 2009). We focus in this study on the interaction of evaporative and radiative cooling with sedimentation, and therefore we neglect cloud-top mean shear, shortwave radiative heating, and surface fluxes. The paper is structured as follows. In section 2 we introduce the formulation and discuss relevant nondimensional numbers that characterize sedimentation. Section 3 describes the two simulated cases that are based on observations in the Arctic and in the tropics. The effect of sedimentation on entrainment is analyzed using the integrated-buoyancy evolution equation in section 4. Finally, we summarize in section 5 how the simulation results can answer the questions introduced above. In particular, we propose how to account for sedimentation in the entrainment velocity parameterization presented in De Lozar and Mellado (2015a).

\section{Formulation}

\section{a. Evolution equations}

In this section, we explain how the linearized formulation introduced in De Lozar and Mellado (2015b) has been modified to account for sedimentation. We initially revisit the full formulation presented in Mellado et al. (2010) and De Lozar and Mellado (2014), which accounts for sedimentation. In a second step, we revise the approximations that lead to the linearized formulation and consistently include sedimentation.

\section{1) THE FULL FORMULATION}

The full formulation is based on the conservation of total water and enthalpy. Without loss of generality, the total water fraction $q_{t}$, specific enthalpy $h$, and liquid fraction $q_{\ell}$ are written in terms of the mixing fraction $\chi$, a variable $\psi$ that quantifies diabatic effects due to radiation or sedimentation (Shao et al. 1997; van Zanten and Duynkerke 2002; Malinowski et al. 2008), and the normalized liquid water content $\ell$ :

$$
\begin{aligned}
q_{t} & =q_{t}^{c}+\left(q_{t}^{d}-q_{t}^{c}\right) \chi, \\
h & =h^{c}+\left(h^{d}-h^{c}\right) \chi+c_{p}^{c} T^{c} \psi, \\
q_{\ell} & =q_{\ell}^{c} \ell,
\end{aligned}
$$

where the superscripts $c$ and $d$ refer to the cloud and free dry atmosphere in a cloud-top mixing layer, and $c_{p}$ is the specific heat. The evolution equations for these variables are derived from the conservation equations in the case that all diffusivities are equal (Mellado et al. 2010):

$$
\begin{aligned}
& d \chi / d t=\kappa_{T} \nabla^{2} \chi-\nabla \cdot\left[\left(\chi_{l}-\chi\right) \mathbf{j}_{\mu}\right], \\
& d \psi / d t=\kappa_{T} \nabla^{2} \psi-r(\ell)-\nabla \cdot\left[\left(\psi_{l}-\psi\right) \mathbf{j}_{\mu}\right], \\
& d \ell / d t=\kappa_{T} \nabla^{2} \ell+\left(\partial_{t} \ell\right)_{\mathrm{pha}}-\nabla \cdot\left[\left(\ell_{l}-\ell\right) \mathbf{j}_{\mu}\right],
\end{aligned}
$$


where $\kappa_{T}$ is the thermal diffusivity, $\left(\partial_{t} \ell\right)_{\text {pha }}$ represents the changes of phase due to condensation/evaporation, and $\mathbf{j}_{\mu}$ is the flux of liquid water due to sedimentation or inertial effects (here neglected). The parameters that multiply the sedimentation flux are $\chi_{l}=\left(1-q_{t}^{c}\right)\left(q_{t}^{d}-q_{t}^{c}\right)^{-1}$, $\ell_{l}=\left(q_{\ell}^{c}\right)^{-1}$, and $\psi_{l}=\left(c_{p}^{c} T^{c}\right)^{-1}\left[\left(h_{l}-h^{c}\right)-\left(h^{t}-h^{c}\right) \chi_{l}^{-1}\right]$, where $h_{l}$ is the water specific latent heat of evaporation. The longwave radiative forcing $r(\ell)$ is based on the onedimensional approximation described by Larson et al. (2007), and it is given by Eq. (A1) in the appendix. Equations (2) are completed by the Navier-Stokes equations in the Boussinesq approximation and by the thermal equation of state that provides the buoyancy, $b=g\left(\rho-\rho^{c}\right) / \rho^{c}$, where $\rho$ is the density.

The sedimentation flux is calculated by assuming that the DSD is described by a lognormal probability distribution function and that the droplet settling velocity is in the Stokes limit (De Lozar and Mellado 2014):

$$
\mathbf{j}_{\mu}=-u_{s} q_{\ell}^{c} \exp \left[5\left(\log \sigma_{g c}\right)^{2}\right] \ell^{5 / 3} \mathbf{e}_{z},
$$

where $\sigma_{g c}$ is the geometric standard deviation of the DSD. The velocity $u_{s}$ is the settling velocity for droplets with radius $r_{c}=\left(3 \rho q_{\ell}^{c}\right)^{1 / 3}\left(4 \pi \rho_{1} N_{d}\right)^{-1 / 3}\left(\rho_{l}\right.$ is the mass density of the liquid), and in the Stokes limit it can be approximated as $u_{s}=a r_{c}^{2}$, with $a=1.19 \times 10^{8} \mathrm{~m}^{-1} \mathrm{~s}^{-1}$ (Rogers and Yau 1989).

\section{2) THE LINEARIZED FORMULATION}

The buoyancy in the linearized formulation is calculated using the Taylor expansions of the thermal equation of state and of the caloric equation of state around the cloud reference state. In this limit, $b$ is a linear function of $\chi, \psi$, and $\ell$. Furthermore, the normalized liquid water content $\ell$ is calculated under the assumption of thermodynamic equilibrium, which avoids solving the evolution equation for $\ell$ [Eq. (2c)]. The liquid water content equilibrium equation is simplified using the Taylor expansion for the saturated vapor content. The resulting liquid and buoyancy functions are

$$
\begin{aligned}
& \ell=f(\xi, \varepsilon)=\varepsilon \ln [\exp (\xi)+1], \quad \xi=1-\chi / \chi_{s}-\psi / \psi_{s}, \\
& b=\left[\chi\left(\frac{1+D}{1-\chi_{s}}\right)+(\ell-1)\left(\frac{D+\chi_{s}}{1-\chi_{s}}\right)\right] \Delta b+\psi g,
\end{aligned}
$$

where $D, \chi_{s}$, and $\psi_{s}$ are constants that depend on the inversion properties, $\Delta b=b^{d}-b^{c}, \xi$ defines the cloud boundary at $\xi=0$, and $\varepsilon$ is a small numerical factor. The function $f$ tends to the piecewise linear function introduced by Bretherton (1987) in the limit $\varepsilon \rightarrow 0$, but it has a finite second-order derivative of order $1 / \varepsilon$, which regularizes the numerical calculations.
For typical atmospheric conditions $\chi_{l} \gg \chi, \psi_{l} \gg \psi$, and $\ell_{l} \gg \ell$, which allows for neglecting the linear terms $(\chi, \psi, \ell)$ that multiply the sedimentation flux in Eqs. (2). Consistent with these approximations, $\psi_{l}$ is calculated assuming that the water specific latent heat of evaporation is constant $\left(h_{l} \simeq h_{l}^{c}\right)$. The accuracy of these approximations is similar to the accuracy in the approximations that lead to the linearized formulation $(\sim 4 \%)$. The resulting formulation has been implemented using the numerical algorithms described in previous publications (Mellado et al. 2010).

Previous studies analyzed the entrainment velocity from the integral analysis of the buoyancy evolution equation, although this equation is not explicitly solved [see Stevens (2002) for a review]. Taking into account all the approximation described above, the evolution equation for the buoyancy reads as follows:

$$
d b / d t=\kappa_{T} \nabla^{2} b-s_{\mathrm{rad}}-s_{\mathrm{eva}}+\nabla \cdot\left[g \mathbf{j}_{\mu}\right]+C(\varepsilon),
$$

where $s_{\text {rad }}$ and $s_{\text {eva }}$ are the buoyancy sources due to radiative and evaporative cooling provided by Eqs. (A3a) and (A3b). Sedimentation introduces a new buoyancy flux term $g \mathbf{j}_{\mu}$ that is investigated in the next sections. The function $C(\varepsilon)$ is a correction factor that appears because of the smoothing introduced by the liquid function $f(\xi, \varepsilon)$, and it is given by Eq. (A3c). In the limit $\varepsilon \rightarrow 0$ the correction factor vanishes: $C(\varepsilon \rightarrow 0) \rightarrow 0$. Small $\varepsilon$ values produce small $C(\varepsilon)$, but they also introduce numerical errors in the calculations of $s_{\text {eva }}$ and make the code less stable when keeping the same resolution $\Delta x / \eta$ (where $\Delta x$ is the grid spacing and $\eta$ is the Kolmogorov scale), as a result of sharper gradients in the buoyancy and liquid fields. We decided to use $\varepsilon=1 / 16$, which is close enough to the limit $\varepsilon \rightarrow 0$ in simulations without sedimentation for a resolution parameter $\Delta x / \eta \lesssim 2.0$ (Mellado et al. 2009) and provides reliable values for $s_{\text {eva }}$. When including sedimentation, we observe that $\varepsilon=1 / 16$ produces an integrated correction term equal to $10 \%$ of the sedimentation buoyancy flux [ $\left.C(1 / 16) d V \sim 0.1 g\left|j_{\mu}\right|\right]$.

\section{b. Nondimensional numbers}

Longwave radiation scales most flow statistics in a cloud-top mixing layer driven by radiative and evaporative cooling (De Lozar and Mellado 2015b). The scales that characterize the radiative forcing are the buoyancy flux $B_{0}=\left(F_{0} g\right)\left(\rho c_{p}^{c} T^{c}\right)^{-1}$, where $F_{0}$ is the cloud-top energy radiative flux, and the extinction length $\lambda$. The buoyancy flux $B_{0} \sim 10^{-3} \mathrm{~m}^{2} \mathrm{~s}^{-3}$ characterizes the injection of turbulent kinetic energy at the larger scales, and thus it scales the energy dissipation rate. The extinction length, $\lambda \sim 15 \mathrm{~m}$, characterizes the region that is 
cooled by radiative cooling, and approximately matches the length scale of the eddies that are relevant for entrainment for relatively flat cloud tops (De Lozar and Mellado 2015b; Gerber et al. 2013). Therefore, the velocity $U_{0}=\left(B_{0} \lambda\right)^{1 / 3}$ and buoyancy $b_{0}=B_{0} U_{0}^{-1}$ roughly characterize the entrainment eddies. When investigating the integrated-buoyancy equation, it is useful to introduce the parameter $\beta$ that scales the integrated-buoyancy source due to radiation: $S_{\mathrm{rad}}=\int_{0}^{\infty}\left\langle S_{\mathrm{rad}}\right\rangle d z=\beta B_{0}$, where the angle brackets mean horizontal averages. In this section we use these radiative scales to derive two settling numbers that characterize sedimentation.

Settling numbers are usually defined as a ratio between a sedimentation velocity and a reference velocity that characterizes the flow. This choice is justified because a so-defined settling number scales the effect of sedimentation on the liquid dynamics in Eq. (2c). Since our focus is on entrainment, we choose the velocity $U_{0}$ that characterizes entrainment eddies as the reference velocity in the settling number definition:

$$
\mathrm{Sv}_{0}=\frac{u_{s} \exp \left[5\left(\log \sigma_{g c}\right)^{2}\right]}{U_{0}},
$$

where the factor $\exp \left[5\left(\log \sigma_{g c}\right)^{2}\right]$ is included because it scales the settling velocity in all the evolution equations. Alternatively, we could use other reference velocities from the range that characterizes a turbulent flow, from the Kolmogorov velocity $w_{\eta}$ up to the integral velocity scale $w^{*}$ [see Eq. (9)]. Discerning which velocity scale is more relevant is, however, difficult, given the relatively limited scale separation that we can achieve: for a simulation domain of size $L$, the scale separation is $L / \eta \sim O\left(10^{3}\right)$ for the length, but only $w^{*} / w_{\eta} \sim O(10)$ for the velocity (assuming a Richardson cascade with inertial scaling).

We define a second settling number as the ratio between the buoyancy fluxes associated with sedimentation and radiative cooling at cloud top:

$$
\mathrm{Sv}_{b}=\frac{g\left|\mathbf{j}_{\mu}^{c}\right|}{\beta B_{0}}=\frac{u_{s} \exp \left[5\left(\log \sigma_{g c}\right)^{2}\right] q_{\ell}^{c} g}{\beta B_{0}}=\operatorname{Sv}_{0} \frac{g q_{\ell}^{c}}{\beta b_{0}},
$$

where $\mathbf{j}_{\mu}^{c}$ is the sedimentation flux in the cloud layer, where $\ell \equiv 1$. The settling number $\mathrm{Sv}_{b}$ scales the effect of sedimentation on the buoyancy dynamics in Eq. (5).

Once the initial conditions are sufficiently forgotten, averaged statistics in a cloud-top mixing layer driven by evaporative and radiative cooling without sedimentation depend on the integral scale of the flow $z^{*}$ and five nondimensional numbers (De Lozar and Mellado 2015a). Sedimentation introduces two additional constants in the evolution equations [Eqs. (2)], implying that two nondimensional numbers are needed to account for sedimentation effects. Using the settling numbers introduced above, the complete set of nondimensional numbers is as follows:

$$
\left\{\mathrm{Re}_{0}, \mathrm{Ri}_{0}, D, \chi_{s}, \beta, \mathrm{Sv}_{0}, \mathrm{~Sv}_{b}\right\},
$$

where $\mathrm{Ri}_{0}=\Delta b / b_{0}$ is a bulk Richardson number, and $\operatorname{Re}_{0}=U_{0} \lambda / \nu$ is a reference Reynolds number ( $\nu$ is the air kinematic viscosity). The parameters $D$ and $\chi_{s}$ fully characterize the water condensation and the evaporative cooling associated with it (Siems and Bretherton 1992), and the combination $\left(1+D / \chi_{s}\right)$ characterizes the potential of the cloud top to produce evaporative cooling (De Lozar and Mellado 2015b).

\section{Simulations setup}

\section{a. Reference cases}

The first reference case is based on the measurements from the research flight 11 from the Vertical Distribution of Ice in Arctic Clouds (VERDI) campaign in the north of Canada (Klingebiel et al. 2015). This case is characterized by a relatively wet inversion and low liquid water content $\left(q_{t}^{d}=2.4 \mathrm{~g} \mathrm{~kg}^{-1}, T^{d}=272.65 \mathrm{~K}, q_{t}^{c}=3.15 \mathrm{~g} \mathrm{~kg}^{-1}\right.$, $T^{c}=268.15 \mathrm{~K}$, and $\left.q_{\ell}^{c}=0.25 \mathrm{~g} \mathrm{~kg}^{-1}\right)$, which implies a low potential for evaporation $\left(D / \chi_{s}+1=0.45\right)$. The droplet number density is $N_{d}=70 \mathrm{~cm}^{-3}$. The second reference case is based on the measurements from the research flight RF01 from the DYCOMS II campaign close to the California coast (Stevens et al. 2005). This case is characterized by a relatively dry inversion and higher liquid water content $\left(q_{t}^{d}=1.5 \mathrm{~g} \mathrm{~kg}^{-1}, T^{d}=292.25 \mathrm{~K}\right.$, $q_{t}^{c}=9 \mathrm{~g} \mathrm{~kg}^{-1}, T^{c}=283.75 \mathrm{~K}$, and $\left.q_{\ell}^{c}=0.5 \mathrm{~g} \mathrm{~kg}^{-1}\right)$, which implies a higher potential for evaporative cooling $\left(D / \chi_{s}+1=1.34\right)$. The droplet number density, $N_{d}=$ $140 \mathrm{~cm}^{-3}$, is taken from van Zanten et al. (2005). In both cases we assume a radiative extinction length $\lambda=15 \mathrm{~m}$, which is close to the value estimated by Larson et al. (2007) for the DYCOMS II case.

\section{b. Simulation parameters}

All simulations are defined by the combination of nondimensional numbers introduced in the previous section and provided in Table 1 . The numbers $\left\{\mathrm{Ri}_{0}, D, \chi_{s}, \beta\right\}$ are calculated from the inversion properties. The other nondimensional numbers are described below.

The settling number based on the velocity is approximated by $\mathrm{Sv}_{0} \simeq 0.04 \exp \left[5\left(\log \sigma_{g c}\right)^{2}\right]$ for both reference cases. The DSD width is typically in the interval $1<\sigma_{g c}<1.5$ and can be chosen to adjust the 
TABLE 1. The first seven columns present the parameters that define each simulation: evaporative cooling is defined by the parameters $D, \chi_{s}$, and $\beta$; the radiative cooling is defined by a reference Richardson number $\mathrm{Ri}_{0}$; the viscous forces are characterized by a reference Reynolds number $\mathrm{Re}_{0}$; and sedimentation is characterized by two settling numbers $\mathrm{Sv}_{0}$ and $\mathrm{Sv}_{b}$. The eighth column shows the domain size in dimensions of the extinction length (typically $\lambda=15 \mathrm{~m}$ ), differentiating between the horizontal and the vertical extension. The ninth column represents the number of points of the numerical grid. The last column indicates the observational campaign that motivated each simulation, where the asterisk denotes cases in which sedimentation is not consistent with the cloud thermodynamic properties (see text). The simulations run for $\sim 4000$ time steps for the cases with $\mathrm{Re}_{0}=400$, and for $\sim 5500$ time steps for the cases with $\mathrm{Re}_{0}=800$.

\begin{tabular}{ccccclllll}
\hline \hline$D$ & $\chi_{s}$ & $\beta$ & $\mathrm{Ri}_{0}$ & $\mathrm{Re}_{0}$ & $\mathrm{~Sv}_{0}$ & $\mathrm{~Sv}_{b}$ & Domain size & Numerical grid & Campaign \\
\hline-0.11 & 0.20 & 0.71 & 28.5 & 400 & 0 & 0 & $(60 \lambda)^{2} \times 30 \lambda$ & $(2048)^{2} \times 1024$ & VERDI \\
-0.11 & 0.20 & 0.71 & 28.5 & 800 & 0 & 0 & $(54 \lambda)^{2} \times 27 \lambda$ & $(3072)^{2} \times 1536$ & VERDI \\
-0.11 & 0.20 & 0.71 & 28.5 & 400 & 0.0425 & 0.024 & $(60 \lambda)^{2} \times 30 \lambda$ & $(2048)^{2} \times 1024$ & VERDI \\
-0.11 & 0.20 & 0.71 & 28.5 & 400 & 0.1 & 0.059 & $(60 \lambda)^{2} \times 30 \lambda$ & $(2048)^{2} \times 1024$ & VERDI \\
-0.11 & 0.20 & 0.71 & 28.5 & 800 & 0.1 & 0.059 & $(54 \lambda)^{2} \times 27 \lambda$ & $(3072)^{2} \times 1536$ & VERDI \\
-0.11 & 0.20 & 0.71 & 28.5 & 400 & 0.1 & 0 & $(60 \lambda)^{2} \times 30 \lambda$ & $(2048)^{2} \times 1024$ & VERDI* \\
0.031 & 0.09 & 0.535 & 41.5 & 400 & 0 & 0 & $(60 \lambda)^{2} \times 30 \lambda$ & $(2048)^{2} \times 1024$ & DYCOMS II \\
0.031 & 0.09 & 0.535 & 41.5 & 800 & 0 & 0 & $(54 \lambda)^{2} \times 27 \lambda$ & $(3072)^{2} \times 1536$ & DYCOMS II \\
0.031 & 0.09 & 0.535 & 41.5 & 400 & 0.0425 & 0.068 & $(60 \lambda)^{2} \times 30 \lambda$ & $(2048)^{2} \times 1024$ & DYCOMS II \\
0.031 & 0.09 & 0.535 & 41.5 & 800 & 0.0425 & 0.068 & $(54 \lambda)^{2} \times 27 \lambda$ & $(3072)^{2} \times 1536$ & DYCOMS II \\
0.031 & 0.09 & 0.535 & 41.5 & 400 & 0.1 & 0.15 & $(60 \lambda)^{2} \times 30 \lambda$ & $(2048)^{2} \times 1024$ & DYCOMS II \\
0.031 & 0.09 & 0.535 & 41.5 & 800 & 0.1 & 0.15 & $(54 \lambda)^{2} \times 27 \lambda$ & $(3072)^{2} \times 1536$ & DYCOMS II \\
0.031 & 0.09 & 0.535 & 41.5 & 400 & 0.1 & 0 & $(60 \lambda)^{2} \times 30 \lambda$ & $(2048)^{2} \times 1024$ & DYCOMS II* \\
- & 0.09 & - & 57 & 400 & 0 & 0 & $(60 \lambda)^{2} \times 30 \lambda$ & $(2048)^{2} \times 1024$ & SMOKE \\
- & 0.09 & - & 57 & 400 & 0.1 & 0 & $(60 \lambda)^{2} \times 30 \lambda$ & $(2048)^{2} \times 1024$ & SMOKE \\
\hline
\end{tabular}

sedimentation strength. Each reference case is simulated in three limits: negligible sedimentation $\left(\mathrm{Sv}_{0}=0\right)$, a narrow DSD $\left(\sigma_{g c}=1.1, \mathrm{~Sv}_{0}=0.0425\right)$ that is similar to the monodisperse DSD, and a broad DSD $\left(\sigma_{g c}=1.54\right.$, $\left.\mathrm{Sv}_{0}=0.1\right)$. The settling numbers based on the buoyancy flux $\mathrm{Sv}_{b}$ are calculated from $\mathrm{Sv}_{0}$ and from the cloud-top thermodynamical properties.

The smallest length scale of a turbulent flow is the Kolmogorov scale, which in DNS is determined by the viscosity (as quantified by $\mathrm{Re}_{0}$ ). To investigate the influence of the smallest scales on the simulation results, we run each simulation with two different reference Reynolds numbers, $\mathrm{Re}_{0}=400$ and $\mathrm{Re}_{0}=800$, which correspond to $\eta \simeq 25$ and $\eta \simeq 15 \mathrm{~cm}$, respectively (as compared to $\eta \simeq 1 \mathrm{~mm}$ in atmospheric conditions).

We have simulated four idealized cases to explore how sedimentation behaves in extreme conditions. The two cases denoted by an asterisk in Table 1 correspond to the limit in which the sedimentation tendency is included on the liquid evolution equation, but not on the buoyancy evolution equation. This is achieved by choosing $\chi_{l}$ and $\psi_{l}$ such that $\ell_{l}=\left(q_{\ell}^{c}\right)^{-1}$ and $b_{l}=0\left[b_{l}\right.$ is the prefactor that multiplies the sedimentation flux in Eq. (5), and for the more realistic cases $b_{l}=g$ ]. The objective of the two SMOKE simulations is to investigate the effect of sedimentation on the radiative forcing, when evaporative cooling and mass-loading effects on the buoyancy are neglected. In these simulations, $(1-\chi)$ represents a weightless smoke field that determines the radiative forcing, and $\psi$ captures the temperature variations induced by radiative cooling. The smoke field plays a similar role for radiation to the liquid in the cloud simulations, and it is driven by the same sedimentation tendency $\left[\chi_{l}=-\left(q_{\ell}^{c}\right)^{-1}\right]$. Mass-loading effects of the smoke field and evaporative cooling are neglected so that buoyancy is uniquely determined by the temperature field $(b=\psi g)$. Since the temperature field does not sediment $\left(\psi_{l}=0\right)$, this assumption implies that there is no buoyancy flux associated to sedimentation $\left(\mathrm{Sv}_{b}=0\right)$. To obtain a similar forcing to the more realistic simulations, radiative cooling is calculated from the liquid field $\ell$ provided by Eq. (4a), which in this case is independent of the temperature field $\left[\left(\psi_{s}\right)^{-1}=0\right]$.

\section{c. Simulations procedure}

Simulations in a cloud-top mixing layer start from a small perturbation in the otherwise zero-velocity field. The flow develops from the cloud top, forming an inverse boundary layer that propagates toward inner parts of the cloud. The size of the boundary layer is well approximated by $\sim 2 z^{*}$, where $z^{*}$ is the integral scale of the in-cloud turbulence defined in De Lozar and Mellado (2015b). The fastest flow velocities are characterized by the integral velocity:

$$
w^{*}=\left(\int\left\langle w^{\prime} b^{\prime}\right\rangle d z\right)^{1 / 3}
$$


which grows as $w^{*} \sim\left(z^{*}\right)^{1 / 3}$. Our past investigations have shown that many in-cloud flow statistics follow scalings typical of a turbulent convective boundary layer when $z^{*} \gtrsim 6 \lambda$, which means that these statistics only depend on $z^{*}$ and on a reference flux [like in the dry convective boundary layer (Deardorff 1970)]. We identify this state with a quasi-steady state, in which also many entrainment statistics asymptotically approach a constant value.

When including sedimentation, the strategy described above leads to a longer transient to the quasisteady state. We observe that the cloud boundary (defined by the liquid water jump) departs from the inversion zone (defined by the buoyancy jump), and it takes a relatively long time for the cloud boundary to return to the inversion zone. The mismatch between inversion and cloud boundary appears at the beginning of the simulations, when flow velocities are comparable to the sedimentation velocity. To minimize this spurious behavior, we initiate all simulations without the sedimentation tendencies until the cloud is close to the quasi-steady state, at $z^{*} \sim 4.5 \lambda$. Typical velocities of the flow at this stage are of order $w^{*} \sim 0.5 \mathrm{~m} \mathrm{~s}^{-1}$, which is much higher than typical sedimentation velocities. In our simulations, it takes 1000 (1800) time steps to reach this state for $\mathrm{Re}_{0}=$ 400 (800). After reaching this state, we include the sedimentation tendencies in the calculations. We observe that the flow then reaches the quasi-steady regime at $z^{*} \sim 8 \lambda$ (e.g., as seen in Fig. 2a). We have used $50 \%$-larger domains in each direction when compared to our previous simulations without sedimentation (see Table 1), which allow us to reach larger integral scales and longer simulation times.

\section{Results}

\section{a. The entrainment velocity}

The entrainment velocity is defined following De Lozar and Mellado (2015b):

$$
\begin{aligned}
w_{e} \Delta b & =-\frac{d}{d t} \int_{z_{i}}^{\infty}\langle b\rangle d z-w_{e}^{\mathrm{diff}} \Delta b \\
& =\left(\frac{d z_{i}}{d t}-w_{e}^{\mathrm{diff}}\right) \Delta b+\frac{d}{d t}\left[\int_{z_{i}}^{\infty}(\Delta b-\langle b\rangle) d z\right],
\end{aligned}
$$

where $z_{i}$ is an inversion point, $\Delta b$ is defined after Eq. $(4 b), w_{e}^{\text {diff }}$ is the entrainment velocity induced by diffusive effects associated with the buoyancy reversal instability (BRI), and the last integral in the rhs accounts for the deformation of the inversion. Equation (10)

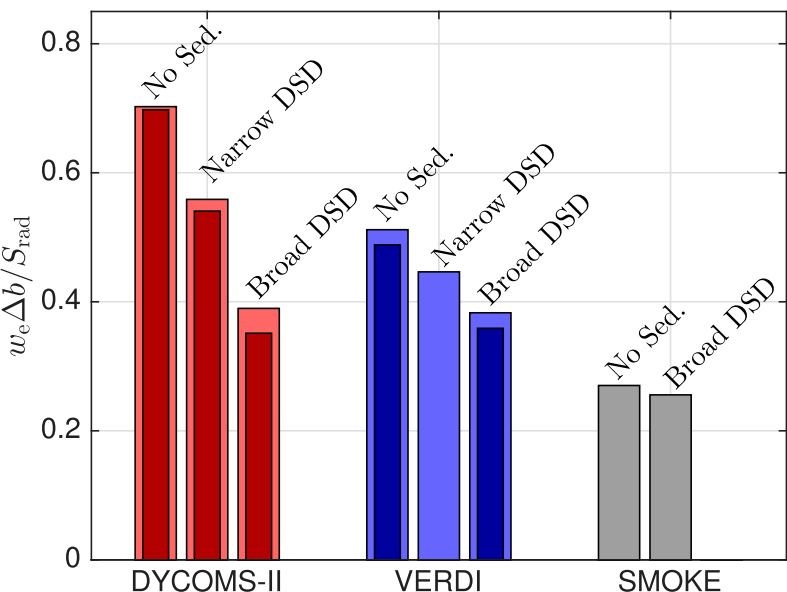

FIG. 1. Entrainment velocity averaged for the time in which the integral length scale is in the interval $9<z^{*} / \lambda<11$. The strength of the sedimentation tendency can be adjusted by assuming different DSD widths. The labels "No Sed.", "Narrow DSD," and "Broad DSD" correspond to simulations with settling numbers $\mathrm{Sv}_{0}=0$, 0.0425 , and 0.1 , respectively. Wide bars correspond to simulations with $\mathrm{Re}_{0}=400$ and narrow bars to $\mathrm{Re}_{0}=800$. Notice how the sensitivity of the entrainment velocity to increasing sedimentation strongly depends on the case.

recovers the traditional definition, $w_{e}=d z_{i} / d t$, when diffusive and deformation effects are neglected. We define the inversion point as the level of neutral buoyancy: $\langle b\rangle\left(z_{i}\right)=0$. One implication of this choice is that the entrainment velocity from Eq. (10) is proportional to the volume-integrated evaporative cooling [see Fig. 3a and De Lozar and Mellado (2015b)], which justifies our inversion point and entrainment velocity definitions. The inversion point divides the simulation domain into the entrainment zone $\left(z>z_{i}\right)$ and the cloud bulk region $\left(z<z_{i}\right)$.

The diffusive velocity $w_{e}^{\text {diff }}$ is negligible for atmospheric conditions, but not for the viscosities we can afford in our simulations $\left[w_{e}^{\text {diff }} \sim 0.2 w_{e}\right.$ in De Lozar and Mellado (2015b)]. The diffusive velocity is estimated with the scalings presented in Mellado (2010). Estimates of the inversion thickness suggest that these scalings are valid up to deviations of $\sim 20 \%$ when sedimentation is included.

Figure 1 shows the averaged entrainment velocity in the interval $9 \lambda<z^{*}<11 \lambda$ for the simulations based on the VERDI and DYCOMS II campaigns and for the SMOKE cloud. The entrainment velocity varies by $\sim 5 \%$ when doubling the reference Reynolds number $\mathrm{Re}_{0}$, suggesting that viscous effects are mostly captured by $w_{e}^{\text {diff. }}$. The simulations also show that the entrainment velocity significantly decreases as the settling parameter $\mathrm{Sv}_{0}$ is increased: sedimentation reduces the entrainment velocity by $20 \%$ when assuming a narrow DSD and by 
$50 \%$ when assuming a broad DSD for the DYCOMS II case.

Figure 1 also shows that the reduction of the entrainment velocity by sedimentation strongly depends on the thermodynamical properties of the inversion. When comparing the cases with no sedimentation to the cases with a broad DSD, we observe that sedimentation reduces $w_{e}$ by $50 \%$ in the DYCOMS II simulations, by $25 \%$ in the VERDI simulations, and by less than $5 \%$ in the SMOKE simulations. The next sections are dedicated to explain this behavior.

\section{b. Integral analysis of the buoyancy evolution equation}

The entrainment velocity defined by Eq. (10) can be calculated from integrating the buoyancy evolution equation, Eq. (5), with the assumption of a negligible correction term $[C(\varepsilon)=0]$ :

$$
-\frac{d}{d t} \int_{z_{i}}^{\infty}\langle b\rangle d z=\left(w_{e}+w_{e}^{\text {diff }}\right) \Delta b=\underbrace{-\left\langle w^{\prime} b^{\prime}\right\rangle_{z_{i}}+\kappa_{\mathrm{T}}\left\langle\frac{\partial b}{\partial z}\right\rangle_{z_{i}}+\int_{z_{i}}^{\infty}\left\langle s_{\text {rad }}\right\rangle d z}_{w_{e}^{\text {rad }} \Delta b}+\underbrace{\int_{z_{i}}^{\infty}\left\langle s_{\text {eva }}\right\rangle d z}_{\left(w_{e}^{\text {eva }}+w_{e}^{\text {diff }}\right) \Delta b}-\underbrace{\left\langle\left|\mathbf{j}_{\mu}\right| g\right\rangle_{z_{i}}}_{w_{e}^{\text {sed }} \Delta b},
$$

which provides a partition of the entrainment velocity into three terms that, to a leading order, are related to radiative cooling, evaporative cooling, and sedimentation. The buoyancy flux at the inversion point:

$$
F=\left\langle w^{\prime} b^{\prime}\right\rangle_{z_{i}}-\kappa_{T}\left\langle\frac{\partial b}{\partial z}\right\rangle_{z_{i}}
$$

is incorporated into the radiative cooling contribution to the entrainment velocity following our past studies on cloud-top mixing layers (De Lozar and Mellado 2013, 2015b), which show that $F$ scales with the radiative forcing. This might not be appropriate when other turbulence sources, like shear or surface fluxes, are also considered.

When evaluating the entrainment velocities $w_{e}^{x x}$ (where $x x$ is the forcing), we have to face a common problem. While the total buoyancy integrated sources $S_{x x}=\int_{0}^{\infty}\left\langle s_{x x}\right\rangle d z$ are known to a good approximation, we know with much less precision the integrals from $z_{i}$ to $\infty$ that appear in Eq. (11). This has motivated us to define efficiencies of the following generic form:

$$
\alpha_{x x}=\frac{\int_{z_{i}}^{\infty}\left\langle s_{x x}\right\rangle d z}{\int_{0}^{\infty}\left\langle s_{x x}\right\rangle d z} .
$$

The efficiencies quantify the fraction of the forcing that "directly" enhances the entrainment velocity, in contraposition to the cooling of the cloud bulk region $\left[\int_{0}^{z_{i}}\left\langle s_{x x}\right\rangle d z\right]$, which can contribute only indirectly to the entrainment through the buoyancy flux $F$ by intensifying the in-cloud turbulence. When the forcing is the radiative cooling, $\alpha_{\text {rad }}$ accounts for the direct radiative cooling induced by droplets in the inversion layer, as introduced initially by Lilly (1968) and later by Lock
(1998) and Moeng (2000). When the forcing is the evaporative cooling, $\alpha_{\text {eva }}$ is equal to the efficiency introduced in De Lozar and Mellado (2015a) in the inviscid limit. In this section, we explore how sedimentation modifies the different contributions to the entrainment velocity in the integrated-buoyancy evolution equation, Eq. (11), for which we make use of the efficiencies provided by Eq. (13).

\section{1) THE SEDIMENTATION EFFECT THROUGH RADIATION}

The distribution of longwave radiative cooling is mostly determined by the liquid water field and by the DSD at the stratocumulus top. Sedimentation alters the liquid water dynamics and, consequently, can change the way that radiative cooling promotes entrainment. We investigate this mechanism by analyzing the term $w_{e}^{\mathrm{rad}} \Delta b$ of the entrainment velocity equation, Eq. (11), that scales with the radiative forcing.

Cloud-top mixing layers and not-very-thin stratocumuli behave as black bodies. As a consequence, the integrated radiative-cooling buoyancy source remains independent of the cloud dynamics:

$$
S_{\mathrm{rad}}=\int_{0}^{\infty}\left\langle s_{\mathrm{rad}}\right\rangle d z=\beta B_{0} .
$$

Combining Eqs. (11), (12), and (14) with the definition for the efficiency, Eq. (13), we obtain

$$
w_{e}^{\mathrm{rad}} \Delta b=-F-\alpha_{\mathrm{rad}} \beta B_{0},
$$

which shows that sedimentation can alter the radiativecooling contribution to the entrainment velocity through the buoyancy flux $F$ or through the radiative-cooling efficiency $\alpha_{\text {rad }}$ that scales the second term of the equation (usually known as direct cooling). 

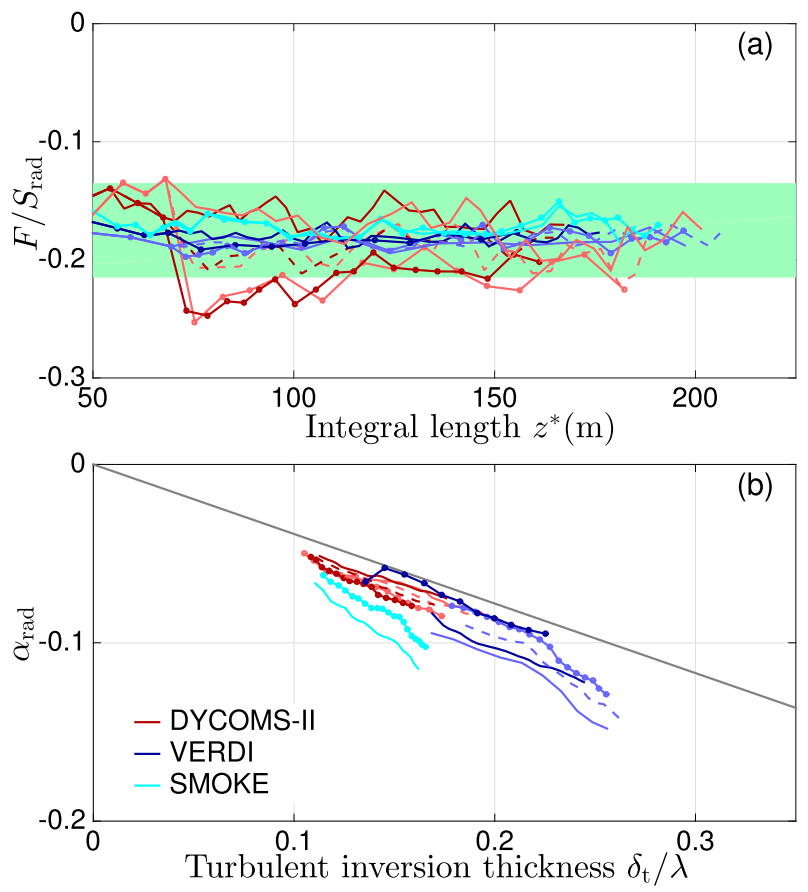

FIG. 2. (a) Buoyancy flux contribution to the radiative entrainment velocity. (b) Efficiency of the radiative cooling to increase the entrainment velocity through direct cooling. The color codes the simulation case: DYCOMS II (red), VERDI (blue), and the SMOKE case (cyan). The line type codes the settling number: $\mathrm{Sv}_{0}=0$ (solid), 0.0425 (dashed), and 0.1 (circles). For VERDI and DYCOMS II, light colors correspond to $\mathrm{Re}_{0}=400$ and darker colors for $\mathrm{Re}_{0}=800$. The green box in (a) and gray line in (b) represent the scalings introduced in De Lozar and Mellado (2013) for simulations with only radiative cooling.

The buoyancy flux $F$ is plotted in Fig. 2a as a function of the boundary layer depth $z^{*}$. We observe that the flux in all simulations asymptotes to $F=(-0.175 \pm 0.04) S_{\text {rad }}$, consistent with simulations with radiative cooling only (De Lozar and Mellado 2013) and with radiative and evaporative cooling (De Lozar and Mellado 2015b). There seems to be little influence of the Reynolds number or of sedimentation.

The radiative cooling efficiency $\alpha_{\text {rad }}$ is plotted in Fig. $2 \mathrm{~b}$ as a function of the turbulent inversion thickness $\delta_{t}=2\left(w^{*}\right)^{2} / \Delta b$, which quantifies the undulation of the cloud-top boundary. The gray line corresponds to the scaling from the simulations with radiative cooling only $\alpha_{\mathrm{rad}}=-0.39 \delta_{t} \lambda^{-1}$, which is also valid for the simulations with radiative and evaporative cooling (De Lozar and Mellado 2013, 2015b). As with the buoyancy flux contribution, we observe that the scaling has a similar accuracy as in previous papers $(\sim 20 \%)$. Changing the Reynolds or settling numbers seems to introduce only small deviations over the scalings proposed above, and therefore we cannot draw a conclusion about a possible dependence on $\mathrm{Sv}_{0}$ or $\mathrm{Re}_{0}$ within $20 \%$ accuracy.
We conclude that the radiative entrainment velocity $w_{e}^{\mathrm{rad}}$ does not appreciably change by introducing sedimentation. This is in agreement with the LES of Bretherton et al. (2007), who observed that only $10 \%$ of the variation of the entrainment velocity by sedimentation can be attributed to changes in the radiative forcing. This explains why the entrainment velocity in the smoke simulation (in which radiation is the only buoyancy source) is the least sensitive to sedimentation.

We employ scaling arguments to explain why the radiative entrainment velocity is mostly insensitive to sedimentation. As explained in section $2 b$, we assume that entrainment eddies are characterized by the length scale $\lambda=15 \mathrm{~m}$, and by the buoyancy flux $B_{0} \sim 1.9 \times 10^{-3} \mathrm{~m}^{2} \mathrm{~s}^{-3}$, which corresponds to a typical entrainment eddy time $t_{0} \sim 50 \mathrm{~s}$. Droplets of radius $R \sim 10 \mu \mathrm{m}$ have a sedimentation velocity $u_{s} \sim 1 \mathrm{~cm} \mathrm{~s}^{-1}$, which implies that they fall $u_{s} t_{0} \sim 0.5 \mathrm{~m}$ because of sedimentation during the entrainment eddy time. This distance is 30 times smaller than the typical radiation length scale $\lambda$, which can explain why sedimentation does not strongly alter the liquid water field in the length scales that are relevant for the bulk radiation model commonly employed in stratocumulus simulations.

\section{2) The SEDIMENTATION EFFECT THROUGH EVAPORATIVE COOLING}

Evaporative cooling happens when droplets mix with dry air from the free atmosphere. Sedimentation removes droplets from the cloud-top region, which might reduce the cloud-top evaporative cooling and weaken entrainment velocity, as proposed by Bretherton et al. (2007). We quantify this effect by analyzing the term of the integral buoyancy evolution equation, Eq. (11), which is associated with the evaporation of droplets, $w_{e}^{\text {eva }} \Delta b$.

Contrary to the integrated-buoyancy source associated with radiation, the integrated-buoyancy source associated with evaporative cooling $S_{\text {eva }}$ depends on the flow dynamics and cannot be calculated solely from the control parameters that describe the inversion zone. However, a relationship between $S_{\text {eva }}$ and the entrainment velocity can be derived when the cloud-top boundary is in a quasi-steady state (De Lozar and Mellado 2015b):

$S_{\text {eva }}=\int_{0}^{\infty}\left\langle s_{\text {eva }}\right\rangle d z=\left(w_{e}+w_{e}^{\mathrm{diff}}\right) \Delta b\left(1+D / \chi_{s}\right)$,

where the parameter $\left(1+D / \chi_{s}\right)$ relates the entrainment of dry air with changes of buoyancy induced by evaporation. Figure 3a shows that the balance provided by Eq. (16) is reached asymptotically in all simulations, an 

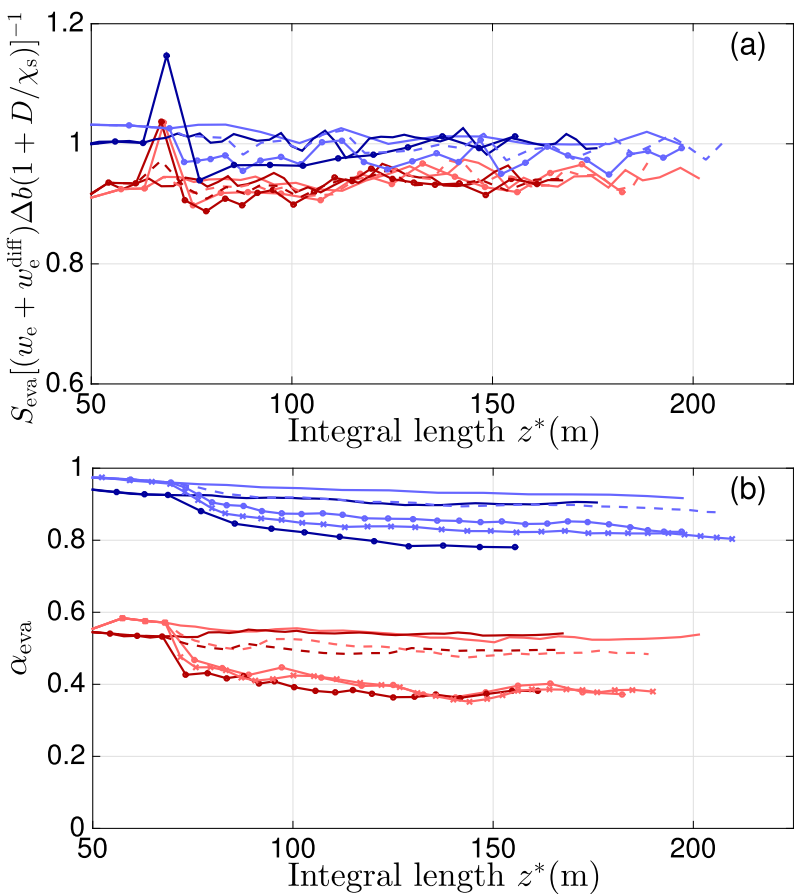

FIG. 3. (a) Total evaporative cooling buoyancy source scaled by the entrainment velocity [see Eq. (16)]. (b) Efficiency of the evaporative cooling to enhance the entrainment velocity. The legend is explained in Fig. 2. Additionally, data from the DYCOMS II* (red crosses) and VERDI* (blue crosses) simulations have been included in (b); see text and Table 1.

indication of the cloud-top boundary reaching the quasisteady state.

The evaporative cooling efficiency is defined in the inviscid limit, after removing the diffusive contributions to the evaporative cooling (De Lozar and Mellado 2015a):

$$
\alpha_{\text {eva }}=\frac{\int_{z_{i}}^{\infty}\left\langle s_{\text {eva }}\right\rangle d z-w_{e}^{\text {diff }} \Delta b}{\int_{0}^{\infty}\left\langle s_{\text {eva }}\right\rangle d z-w_{e}^{\text {diff }} \Delta b\left(1+D / \chi_{s}\right)},
$$

which, in combination with Eq. (16), leads to

$$
w_{e}^{\text {eva }} \Delta b=\alpha_{\text {eva }}\left(1+D / \chi_{s}\right) w_{e} \Delta b .
$$

This equation shows that sedimentation can alter the evaporative cooling contribution for the entrainment velocity by changing the evaporative cooling efficiency $\alpha_{\text {eva. }}$.

Figure $3 \mathrm{~b}$ shows that $\alpha_{\text {eva }}$ depends only mildly on the integral length scale $z^{*}$, suggesting that larger simulations will produce very similar efficiencies. Similarly, we observe that $\alpha_{\text {eva }}$ is independent of $\mathrm{Re}_{0}$ for the DYCOMS II simulations and that it decreases by only $5 \%$ when doubling the viscosity in the VERDI simulations. This result confirms that $w_{e}^{\text {diff }}$ captures the dependence on $\mathrm{Re}_{0}$ and supports the extrapolation of our results to atmospheric conditions where $\mathrm{Re}_{0}$ is much larger (the reason for the remaining 5\% dependence in VERDI is that, while $w_{e}^{\text {diff }}$ captures most viscous effects in the DYCOMS II simulations, we can expect still some effects of viscosity for the VERDI simulations in which there is no BRI and $w_{e}^{\text {diff }}=0$ ).

The evaporative cooling efficiency $\alpha_{\text {eva }}$ decreases with increasing sedimentation parameter. When compared with the simulations with no sedimentation, the efficiency decreases by $\sim 0.04$ for the simulations with $\mathrm{Sv}_{0}=0.0425$ and by 0.1 (VERDI) and by 0.14 (DYCOMS II) for the simulations with $\mathrm{Sv}_{0}=0.1$, which can be translated to a roughly $15 \%-30 \%$ reduction of $w_{e}$ for $\mathrm{Sv}_{0}=0.1$. This means that sedimentation can considerably alter the evaporative dynamics and have a nonnegligible effect on the entrainment velocity.

We have investigated whether the reduction of the evaporative cooling efficiency $\alpha_{\text {eva }}$ is caused by the different liquid dynamics (through $\mathrm{Sv}_{0}$ ) or by the sedimentation flux (through $\mathrm{Sv}_{b}$, as described in the next section). To this end, we have performed two simulations for the DYCOMS II and VERDI campaigns (denoted by an asterisk in Table 1) in which the liquid evolution equation is the same as in the cases with a broad DSD $\left(\mathrm{Sv}_{0}=0.1\right)$, but in which sedimentation does not alter the buoyancy evolution equation $\left(\mathrm{Sv}_{b}=0\right.$, as described in section 3 ). Figure 3 shows that the efficiencies $\alpha_{\text {eva }}$ in the simulations without sedimentation tendencies in the buoyancy equation (crosses in Fig. 3b) are very similar to the simulations with sedimentation tendencies (circles). We conclude that the reduction of the evaporative cooling efficiency is only an effect of the different liquid dynamics, and it has to be characterized by a settling number based on a velocity scale (like $\mathrm{Sv}_{0}$ ).

The significant reduction of the evaporative cooling entrainment velocity by sedimentation is consistent with the idea that evaporative cooling substantially enhances entrainment when droplets evaporate in the entrainment zone defined by the buoyancy profile (De Lozar and Mellado 2015b). According to this idea, the reduction of the entrainment velocity is explained because sedimentation moves the cloud-dry air interface where droplets evaporate away from the entrainment zone. As a consequence, sedimentation increases the fraction of droplets that evaporate in the cloud bulk (usually in the downdrafts regions), where evaporative cooling does not directly enhance the entrainment velocity. This picture also explains why the dynamics that determine the cloud-dry air interface (the liquid dynamics) are more relevant for the evaporative cooling efficiency 
than the additional buoyancy flux associated with sedimentation.

\section{3) ThE SEDIMENTATION BUOYANCY FLUX}

At cloud top, the liquid water content rapidly increases from zero in the free atmosphere to the in-cloud value. Droplets at the cloud-top boundary thus fall into regions with higher liquid water content and leave a lighter air behind them. This appears in Eq. (11) as a positive buoyancy flux into the entrainment zone $w_{e}^{\text {sed }} \Delta b$, which can be seen as a negative contribution to the entrainment velocity.

Analogous to radiative and evaporative cooling, we define the total integrated-buoyancy source due to sedimentation as follows:

$$
\begin{aligned}
S_{\text {sed }} & =-\int_{0}^{\infty} \nabla \cdot\left[\left\langle g \mathbf{j}_{\mu}\right\rangle\right] d z=g\left|\mathbf{j}_{\mu}^{c}\right|=u_{s} q_{\ell}^{c} g \exp \left[5\left(\log \sigma_{g c}\right)^{2}\right] \\
& =\operatorname{Sv}_{b} S_{\text {rad }},
\end{aligned}
$$

which is constant in a cloud-top mixing layer configuration. The cloud-top sedimentation buoyancy flux is characterized by $S_{\text {sed }}$, and its definition can be extended to cloud layers when the lower integration limit of Eq. (19) is placed at the height for which $\left\langle q_{\ell}\right\rangle=q_{\ell}^{c}$.

The sedimentation efficiency is defined by using the generic form provided by Eq. (13):

$\alpha_{\text {sed }}=\frac{\int_{z_{i}}^{\infty} \nabla \cdot\left[\left\langle g \mathbf{j}_{\mu}\right\rangle\right] d z}{\int_{0}^{\infty} \nabla \cdot\left[\left\langle g \mathbf{j}_{\mu}\right\rangle\right] d z}=\frac{g\left\langle\left|\mathbf{j}_{\mu}\right|\right\rangle_{z_{i}}}{g\left|\mathbf{j}_{\mu}^{c}\right|}=\left\langle\ell^{5 / 3}\right\rangle_{z_{i}}$,

where the last equality has been calculated with the sedimentation flux from Eq. (3). The sedimentation efficiency thus crucially depends on the relative position of the inversion point in the cloud-dry air interface, and it is of order 1.

Combining Eqs. (19) and (20) leads to

$$
\begin{aligned}
w_{e}^{\mathrm{sed}} \Delta b & =\alpha_{\mathrm{sed}} S_{\mathrm{sed}}=\alpha_{\mathrm{sed}} u_{s} q_{\ell}^{c} g \exp \left[5\left(\log \sigma_{g c}\right)^{2}\right] \\
& =\alpha_{\mathrm{sed}} \mathrm{Sv}_{b} S_{\mathrm{rad}},
\end{aligned}
$$

which shows that the effect of the sedimentation buoyancy flux on the entrainment velocity scales with $S_{\text {sed }}$ and with a factor of order $1, \alpha_{\text {sed }}$, which depends on the liquid dynamics. Notice that the nondimensional number $\mathrm{Sv}_{b}$ quantifies the relative importance of the buoyancy sedimentation flux with respect to radiative cooling for the entrainment dynamics, as introduced in section 2.

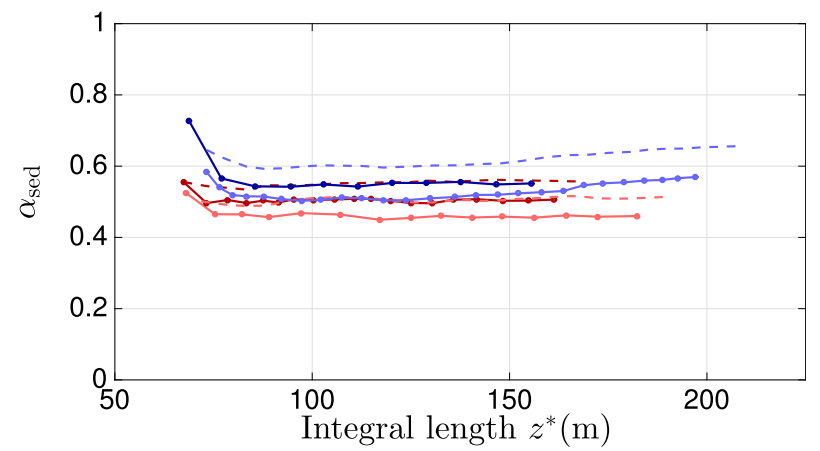

FIG. 4. Efficiency of the buoyancy flux associated with sedimentation to reduce the entrainment velocity as a function of the integral length scale. The legend is explained in Fig. 2.

Figure 4 shows that $\alpha_{\text {sed }}$ does not strongly depend on the integral flow scale, thus suggesting that the larger length scales of the flow are not relevant for this statistic. In all our simulations, the sedimentation efficiency lies in the interval $0.45 \leq \alpha_{\text {sed }} \leq 0.65$, suggesting that this efficiency is mostly independent of the inversion's thermodynamic and radiative properties, $\left\{D, \chi_{s}, \beta, B_{0}, \lambda\right\}$. However, this result needs to be better assessed, given the limited number of cases studied here. Besides, the sedimentation efficiency seems sensitive to the smallest scales of the flow, although moderately, as it increases by $\sim 10 \%$ when doubling $\operatorname{Re}_{0}$ for all cases. Since other statistics converge at the Reynolds numbers of the simulations, we can also expect that $\alpha_{\text {sed }}$ at $\operatorname{Re}_{0}=800$ is close to the limit of the high Reynolds number. Nonetheless, we cannot rule out the possibility that $\alpha_{\text {sed }}$ might reach values close to 1 for atmospheric conditions $\left(\mathrm{Re}_{0} \sim 10^{5}\right)$, which from Eq. (20) would imply that the inversion point is inside the cloud.

We observe that the inclusion of $w_{\text {sed }}$ alone reduces the entrainment velocity by $4 \%(10 \%)$ for VERDI and by $10 \%(25 \%)$ for DYCOMS II, when assuming $\alpha_{\text {sed }}=0.55$ and a narrow (broad) DSD characterized by $\mathrm{Sv}_{0}=0.0425\left(\mathrm{~Sv}_{0}=0.1\right)$. This indicates that the sedimentation buoyancy flux cannot be neglected in the calculations of the entrainment velocity for typical meteorological conditions.

\section{Consequences for atmospheric models}

\section{a. Comparison with LES}

Bretherton et al. (2007) investigated the sensitivity of the entrainment velocity to sedimentation in LES based on the measurements of the research flight RF01 of the DYCOMS II campaign, which is the same case as here. They found that sedimentation reduces the entrainment velocity by $3.5 \%$ for a narrow DSD $\left(\sigma_{g c}=1.2\right)$ and by 
$7 \%$ for a broad DSD $\left(\sigma_{g c}=1.5\right)$. These values are much smaller than in our simulations (reduction of $20 \%$ and $50 \%$ for the narrow and wide DSD, respectively) for very similar cases. Part of this difference is explained by the reduction of cloud-top liquid water content in Bretherton et al. (2007) from $q_{\ell}^{c}=0.5 \mathrm{~g} \mathrm{~kg}^{-1}$ to a quasisteady $q_{\ell}^{c}=0.25 \mathrm{~g} \mathrm{~kg}^{-1}$ during the initial transient so that the sedimentation strength is reduced. To compare the different states, we use the two settling numbers $\mathrm{Sv}_{0}$ and $\mathrm{Sv}_{b}$ introduced in this paper. We estimate that the simulations of Bretherton et al. (2007) with $q_{\ell}^{c}=0.25$ are characterized by the pair $\left(\mathrm{Sv}_{0}=0.03, \mathrm{~Sv}_{b}=0.023\right)$ for the narrow DSD and by $\left(\mathrm{Sv}_{0}=0.059, \mathrm{~Sv}_{b}=0.044\right)$ for the broad DSD. Their simulations with the broad DSD are thus closer to the simulation with a narrow DSD presented in this paper $\left(\mathrm{Sv}_{0}=0.0425, \mathrm{~Sv}_{b}=0.068\right)$, but the reduction of the entrainment velocity is still stronger in the present study ( $20 \%$ vs $7 \%)$.

We also compare our results with the intercomparison study of Ackerman et al. (2009), which is based on the observations of the research flight RF02 from the DYCOMS II campaign with $N_{d}=55 \mathrm{~cm}^{-3}$. These simulations produce a cloud top with a quasi-steady liquid water content $q_{\ell}^{c}=0.5 \mathrm{~g} \mathrm{~kg}^{-1}$, and a reduction of the entrainment velocity by sedimentation of $15 \%$ for a narrow DSD $\left(\sigma_{g c}=1.2\right)$ and of $25 \%$ for a broad DSD $\left(\sigma_{g c}=1.5\right)$. The settling numbers in the narrow DSD case $\left(\mathrm{Sv}_{0}=0.09, \mathrm{~Sv}_{b}=0.135\right)$ are similar to the broad DSD case investigated in this paper for DYCOMS II $\left(\mathrm{Sv}_{0}=0.1, \mathrm{~Sv}_{b}=0.15\right)$, but again the reduction the entrainment velocity is stronger in the present study ( $50 \%$ vs $15 \%)$.

The sensitivity of the entrainment velocity to sedimentation in our study is approximately 3 times larger than in past LES. Part of these differences can be attributed to disparity in the thermodynamic inversion properties that determine the evaporative forcing, but these are not so large as to explain such big differences in the sedimentation response. Our simulations based on the VERDI campaign still show a stronger response to sedimentation [ $25 \%$ for $\left.\left(\mathrm{Sv}_{0}=0.1, \mathrm{~Sv}_{b}=0.059\right)\right]$ than in LES with drier free atmospheres and higher settling numbers. We conjecture that the weak response of LES to sedimentation is because current resolutions $(\Delta z=$ $5 \mathrm{~m}, \Delta x \geq 35 \mathrm{~m}$ ) artificially enhance mixing at meter and submeter scales, with the consequence that numerical diffusion might mask sedimentation effects to some extent. This can partly explain why Caldwell and Bretherton (2009) had to assume a very strong sedimentation $\left(\sigma_{g c}=2\right.$, which implies $\left.\mathrm{Sv}_{0} \sim 0.5\right)$ to obtain realistic values of the entrainment velocity in their stratocumulus LES. The consequence is that future LES will probably show a stronger response to sedimentation as resolution is increased.

\section{b. Parameterization of the entrainment velocity}

In this section, we apply the results from the integral analysis of the buoyancy evolution equation (section 4) for including sedimentation in the entrainment velocity parameterization that was developed in De Lozar and Mellado (2015a). The parameterization is not meant to be definitive, as it is based on the study of only two cases, but it captures the general physics explained in this paper and can be a good basis for future studies.

Combining Eqs. (11), (16), and (17), the entrainment velocity can be written as follows:

$$
w_{e}=\frac{w_{e}^{\mathrm{rad}}-w_{e}^{\mathrm{sed}}}{1-\alpha_{\mathrm{eva}}\left(D / \chi_{s}+1\right)},
$$

where $w_{e}^{\mathrm{rad}}$ is the contribution to the entrainment velocity due to radiative cooling, $w_{e}^{\text {sed }}$ is the direct reduction of the entrainment velocity by sedimentation due to the droplets weight, and $\alpha_{\mathrm{eva}}$ is the evaporative cooling efficiency to enhance the entrainment velocity. Based on the results presented in section 4 and in De Lozar and Mellado (2015a), we propose the following parameterizations for these terms:

$$
\begin{aligned}
w_{e}^{\mathrm{rad}} \Delta b \simeq & {\left[0.175+0.78\left(w^{*}\right)^{2} /(\lambda \Delta b)\right] \beta B_{0}, } \\
w_{e}^{\mathrm{sed}} \Delta b \simeq & 0.55 u_{s} q_{\ell}^{c} g \exp \left[5\left(\log \sigma_{g c}\right)^{2}\right], \\
\alpha_{\mathrm{eva}} \simeq & 0.7-0.3 \tanh \left(2.5 D / \chi_{s}\right) \\
& -u_{s} \exp \left[5\left(\log \sigma_{g c}\right)^{2}\right]\left(B_{0} \lambda\right)^{-1 / 3},
\end{aligned}
$$

which are justified in the next paragraphs.

The radiative contribution to the entrainment velocity $w_{e}^{\mathrm{rad}}$ does not change beyond the statistical convergence $(\sim 10 \%)$ by including sedimentation in our simulations. This trend is confirmed in simulations of a smoke cloud (with no evaporative cooling), in which the entrainment velocity decreases only by $\sim 5 \%$ when imposing a strong sedimentation. We conclude that sedimentation has only a weak influence over the radiative forcing, in agreement with Bretherton et al. (2007), and therefore it is not considered for the parameterization. Equation (23a) recovers the original parameterization of $w_{e}^{\text {rad }}$ for the cases with radiative and evaporative cooling (De Lozar and Mellado 2015a) and for radiative cooling only (De Lozar and Mellado 2013), and it has a similar functional form as entrainment velocity parameterizations that do not consider evaporative cooling in detail (Lock 1998; Moeng et al. 1999) when the cloud top is relatively flat $\left[\left(w^{*}\right)^{2}(\Delta b)^{-1} \ll \lambda\right]$.

Sedimentation promotes a positive buoyancy flux into the cloud top, $u_{s} q_{\ell}^{c} g \exp \left[5\left(\log \sigma_{g c}\right)^{2}\right]$, because of the weight carried by the droplets as they sediment. This 
mechanism reduces the entrainment flux $w_{e} \Delta b$ by $w_{e}^{\text {sed }} \Delta b$, which is a fraction of the aforementioned sedimentation buoyancy flux. The proportionality factor in our simulations is $\alpha_{\text {sed }}=0.55 \pm 0.1$, as in Eq. (23b). However, we notice that $\alpha_{\text {sed }}$ did not converge in our simulations with respect to $\mathrm{Re}_{0}$, even when the resolution is only a few tens of centimeters, and it might be larger for higher resolutions. Besides, $\alpha_{\text {sed }}$ might depend on the thermodynamic properties of the inversion and have different values than in the cases here investigated. This dependence remains to be systematically quantified.

Sedimentation alters the liquid-field dynamics by removing cloud droplets from the entrainment zone. This mechanism reduces the capacity of evaporative cooling to enhance the entrainment velocity, as quantified by $\alpha_{\text {eva }}$. In our simulations, we have observed that (i) this mechanism has the potential to considerably reduce the entrainment velocity and (ii) that the variations of $\alpha_{\text {eva }}$ depend on a settling number based on the sedimentation velocity $\mathrm{Sv}_{\text {ref }}=u_{s} \exp \left[5\left(\log \sigma_{g c}\right)^{2}\right] / U_{\text {ref }}$ and are independent of the sedimentation buoyancy flux. We propose to use a settling number $\mathrm{Sv}_{\mathrm{ref}}=\mathrm{Sv}_{0}$ based on the radiative velocity scale, which is obtained by using $U_{\text {ref }}=U_{0}=\left(B_{0} \lambda\right)^{1 / 3}$. This choice is consistent with the idea that entrainment eddies are characterized by velocity scales similar to the radiative velocity scale (De Lozar and Mellado 2013; Gerber et al. 2013). In our simulations, the deviations of the efficiency due to sedimentation can be quantified as $\left[\alpha_{\text {eva }}\left(\operatorname{Sv}_{0}\right)-\alpha_{\text {eva }}\left(\operatorname{Sv}_{0}=0\right)\right]=(1 \pm 0.5) \operatorname{Sv}_{0}$. Equation. (23c) combines this expression with the functional form for $\alpha_{\mathrm{eva}}\left(\mathrm{Sv}_{0}=0\right)$ that better describes the simulations presented in De Lozar and Mellado (2015a). The large error in the numerical factor $(1 \pm 0.5)$ is explained because this factor is probably not constant and depends on the inversion properties and because maybe the linear relationship is too simple to capture this effect. As occurred with $\alpha_{\text {sed }}$, a more detailed investigation is necessary to clarify this point.

To our knowledge, the only entrainment velocity parameterization that accounts for sedimentation previous to this work is the parameterization of Turton and Nicholls (1987) modified by Bretherton et al. (2007), which we denote as NTB. The parameterization introduced by Eqs. (22) and (23) (here denoted as IA, because of the integral analysis) recovers the same functional form as NTB in the limit $\left(D / \chi_{s}+1\right) \ll \mathrm{Sv}_{0} \ll 1$, in which $\alpha_{\text {eva }} \simeq 1-a^{\prime} \mathrm{Sv}_{0} \simeq$ $\exp \left(-a^{\prime} \mathrm{Sv}_{0}\right)$, and therefore

$$
w_{e} \simeq\left(w_{e}^{\mathrm{dry}}-w_{e}^{\mathrm{sed}}\right)\left[1+a_{2} \Delta_{m} \exp \left(-a_{\mathrm{sed}} u_{s} / U_{\mathrm{ref}}\right)\right],
$$

where $a^{\prime}$ and $a_{\text {sed }}$ are a numerical factors that depend on the DSD width, $w_{e}^{\text {dry }}$ is the entrainment velocity in case of no evaporative cooling, $\Delta_{m}=D+\chi_{s}$ measures the integrated-buoyancy excess due to evaporative cooling, and the parameter $a_{2}$ scales the effect of evaporation on entrainment. While in NTB $a_{2}^{\mathrm{NTB}} \in(15,60)$ is a free parameter, in our parameterization its value is set: $a_{2}^{\mathrm{IA}}=\chi_{s}^{-1}$. In both parameterizations, sedimentation reduces the evaporative cooling enhancement of the entrainment velocity by a factor that depends on a settling number $\mathrm{Sv}_{\text {ref }} \sim u_{s} / U_{\text {ref }}$, as shown by the exponential factor in Eq. (24). The IA parameterization differs from NTB in the choice of the settling number reference velocity $\left(U_{\mathrm{ref}}^{\mathrm{NTB}}=w^{*}\right.$ vs $\left.U_{\mathrm{ref}}^{\mathrm{IA}}=U_{0}\right)$, but, as mentioned in section 2 and in Bretherton et al. (2007), the appropriateness of this choice is difficult to test. A more relevant difference is that the IA parameterization considers a direct effect of sedimentation on the entrainment velocity, which is missing in NTB $\left(w_{e}^{\text {sed,NTB }}=0\right)$.

The IA and NTB parameterizations clearly differ when $\left(D / \chi_{s}+1\right) \sim 1$, as the evaporative cooling enhancement of the entrainment velocity enters very differently in both cases. However, both parameterizations still agree that the evaporative cooling enhancement is reduced due to sedimentation by a factor that depends on a settling number [the exponential factor in NTB and the last term of Eq. (23c) in IA].

\section{c. Sensitivity of the entrainment velocity to the cloud droplet number density}

Our simulations show that sedimentation can significantly reduce the entrainment velocity $(\sim 20 \%$ for DYCOMS II RF01 with $N_{d}=140 \mathrm{~cm}^{-3}$ and a narrow DSD). Since sedimentation crucially depends on the droplets' radii, this result implies that increasing the cloud droplet number density can significantly enhance the entrainment velocity. We explore in this section the sensitivity of the entrainment velocity to cloud droplet number density. This analysis is limited to changes in the entrainment velocity due to sedimentation or radiative cooling. Other microphysical effects, like finite-time evaporation, are not discussed, although they are expected to further enhance the entrainment velocity as the number density is increased (Hill et al. 2008). The basis of the analysis is the investigation of the parameterization introduced by Eqs. (22) and (23).

Sedimentation reduces the cloud-top evaporation and, consequently, the entrainment velocity, as proposed by Bretherton et al. (2007) and Ackerman et al. (2009). In our analysis, we identify this effect with the reduction of the evaporative cooling efficiency, $\alpha_{\mathrm{eva}}$ in Eq. (22), by sedimentation. This reduction is roughly proportional to a settling number based on the settling 
velocity like $\mathrm{Sv}_{0}$ [Eq. (23c)], which is proportional to $r_{c}^{2}$ and therefore to $N_{d}^{-2 / 3}$. For DYCOMS II inversions, we estimate that this effect alone reduces the entrainment velocity with respect to the sedimentation-free case by $5 \%$ for $N_{d}=1000 \mathrm{~cm}^{-3}$, by $15 \%$ for $N_{d}=150 \mathrm{~cm}^{-3}$, and by $30 \%$ for $N_{d}=50 \mathrm{~cm}^{-3}$, when using $\sigma_{g c}=1.2$. Equation (22) shows that the sensitivity of the entrainment velocity to $N_{d}$ through $\alpha_{\text {eva }}$ also depends on the capacity of the inversion to produce evaporative cooling, as quantified by $1+D / \chi_{s}$ (De Lozar and Mellado 2015b). Stratocumuli capped by dry inversions (large $1+D / \chi_{s}$ as in DYCOMS II) are thus expected to be more sensitive to variations in the droplet number density than stratocumuli capped by moist capping inversions (small $1+D / \chi_{s}$ as in VERDI).

In addition to the reduction of evaporation, sedimentation promotes a positive buoyancy flux into the entrainment zone that decreases the entrainment velocity by $w_{e}^{\text {sed }}$, as shown by Eq. (22). The relative importance of this effect is captured by a second settling number that relates the buoyancy fluxes that characterize sedimentation and radiation: $\mathrm{Sv}_{b} \sim 2 \times 10^{3} q_{\ell}^{c} \beta^{-1} \mathrm{~Sv}_{0}$ (using $F_{0}=70 \mathrm{~W} \mathrm{~m}^{-2}$ and $\lambda=15 \mathrm{~m}$ ). Again, this settling number is proportional to $r_{c}^{2}$, and therefore to $N_{d}^{-2 / 3}$, which captures the sensitivity of $w_{e}^{\text {sed }}$ to changing the cloud droplet number density. We estimate that this effect alone reduces the entrainment velocity by $2.5 \%$ for $N_{d}=1000 \mathrm{~cm}^{-3}$, by $9 \%$ for $N_{d}=150 \mathrm{~cm}^{-3}$, and by $17 \%$ for $N_{d}=50 \mathrm{~cm}^{-3}$, when using Eq. (23b) with $\sigma_{g c}=1.2$ and DYCOMS II RF01 conditions. Although clearly related, the entrainment velocity reduction by the sedimentation flux can be quite different to the reduction by altering evaporation. For example, the sedimentation buoyancy flux crucially depends on the cloudtop liquid water content $q_{\ell}^{c}$, and we expect a significant effect of sedimentation on the entrainment velocity when $q_{\ell}^{c}$ is high, even for moderate droplet sizes that imply small $\mathrm{Sv}_{0}$ [although if high $q_{\ell}^{c}$ is associated with small $\sigma_{g c}$ (Geoffroy et al. 2010), this relation might reduce the expected sedimentation buoyancy flux]. Besides, the sedimentation buoyancy flux is independent of the thermodynamical properties of the free atmosphere, which means that changing the cloud droplet number density can still have a significant effect on the entrainment velocity through sedimentation for moist inversions.

In addition to sedimentation, the entrainment velocity is sensitive to varying the cloud droplet number density through the direct radiative cooling (not related to the aerosol direct effect) (Moeng et al. 1999; De Lozar and Mellado 2013). The direct radiative cooling in our parameterization is given by the second term of Eq. (23a), which is inversely proportional to the extinction length $\lambda$. Simple estimates show that the extinction length scales as follows: $\lambda \sim r_{c} \sim N_{d}^{-1 / 3}$ (e.g., Petty 2006). Using this approximation in Eqs. (23a) and (22) implies that the entrainment velocity increases by $\sim 35 \%$ when incrementing $N_{d}$ by a factor of 10 . We conclude that the entrainment velocity sensitivity to $N_{d}$ due to changes in the extinction length can be comparable to the sensitivity induced by sedimentation.

When combining all mechanisms described in this section, we estimate that entrainment velocities for typical cloud droplet number densities over land $\left(N_{d} \sim 300 \mathrm{~cm}^{-3}\right)$ are about $25 \%-50 \%$ larger than over the ocean $\left(N_{d} \sim 50 \mathrm{~cm}^{-3}\right)$, for the same thermodynamical properties of the atmospheric boundary layer. These differences are much larger than in past LES (Bretherton et al. 2007; Ackerman et al. 2009), suggesting that stratocumulus entrainment is more sensitive to the cloud droplet number density than previously thought.

\section{Conclusions}

We use DNS to investigate how sedimentation alters stratocumulus entrainment at length scales that range from the resolved Kolmogorov length scale, $\eta \sim \Delta x=$ $26 \mathrm{~cm}$, to the integral length scale, $z^{*} \sim 150-200$. Varying the Kolmogorov and integral length scales beyond this range produces only mild variations in the entrainment statistics, which indicates that the DNS resolve most length scales that are relevant for the interaction of entrainment with sedimentation. The simulations' results have been used to analyze the integrated-buoyancy evolution equation and to develop a parameterization of the entrainment velocity for entrainment closures in large-scale models.

Sedimentation reduces the entrainment rate by depleting liquid droplets from the entrainment zone. In accordance with Bretherton et al. (2007), we observe that the depletion of liquid water considerably weakens the evaporative cooling enhancement of the entrainment velocity but has a minor effect on the longwave radiative forcing. This last point is confirmed by smokecloud simulations (only driven by radiative cooling) where the entrainment velocity is almost independent of sedimentation.

The reduction of the entrainment velocity by sedimentation in DNS is 3 times larger than previously reported in LES (Bretherton et al. 2007; Ackerman et al. 2009). This discrepancy is investigated by analyzing the integrated-buoyancy evolution equation. The analysis uncovers a new mechanism that significantly reduces the entrainment velocity: the weight of sedimenting droplets promotes a positive buoyancy flux that directly opposes the entrainment flux at the inversion. This 
mechanism is characterized by a settling number based on the buoyancy flux, which differs from the more traditional settling number (based on the velocity) that characterizes the reduction of the evaporative cooling enhancement by sedimentation discussed by Bretherton et al. (2007). This means that two different settling numbers are needed to characterize the reduction of entrainment velocity by sedimentation, as it is reflected in the entrainment velocity parameterization proposed here. Our analysis shows that the dominant mechanisms through which sedimentation reduces entrainment operate at the very thin cloud interface. This could explain why LES at current resolutions, which artificially enhance mixing at meter and submeter scales, produce a weaker response to sedimentation. Since sedimentation is directly related to the cloud droplet number density, this work suggests that stratocumulus entrainment is significantly more sensitive to the cloud droplet number density than previously thought.

Acknowledgments. The source code and initialization files can be downloaded from the Max Planck Society Publication Repository (http://pubman.mpdl.mpg.de/ pubman/faces/viewItemOverviewPage.jsp?itemId=escidoc: 2309103). All data used for this study will be provided upon request to anyone interested. Support from the Max Planck Society through its Max Planck Research Groups program is gratefully acknowledged. We are grateful to Andrew Ackerman and to one anonymous reviewer, who helped to improve the manuscript. Computational time was provided by the Jülich Supercomputing Centre.

\section{APPENDIX}

\section{Supplementary Formulation}

For completeness, we include an appendix with the exact expression for the functions and parameters introduced in this paper. All expressions can be derived from the equations presented in this paper in combination with the formulation introduced in De Lozar and Mellado (2015b). Notice that there is a factor $\left(c_{p}^{c} T^{c}\right)$ difference in the definition of $\psi$ in De Lozar and Mellado (2015b) from the definition used in this publication.

The radiative forcing used in this paper is based on Larson et al. (2007), and it is given by

$r(\ell)=B_{0}(\lambda g)^{-1} \ell \exp \left[-\lambda^{-1} \int_{z}^{z_{\text {top }}} \ell\left(z^{\prime}\right) d z^{\prime}\right]$,

where $z_{\text {top }}$ is the height at the top of the simulation domain.

The parameters $D, \chi_{s}$, and $\beta$ can be written as a function of the thermodynamical properties of the inversion, as presented in De Lozar and Mellado (2015b). Their expressions are, however, complex and will not be repeated here. The parameter $\psi_{s}$ can be written as follows:

$$
\psi_{s}=\Delta b g^{-1}\left(D+\chi_{s}\right)\left(1-\chi_{s}\right)^{-1}(1-\beta)^{-1} .
$$

The sources and correction term in the buoyancy evolution equation, Eq. (5), are

$$
\begin{aligned}
s_{\mathrm{rad}} & =\beta r(\ell) g, \\
s_{\mathrm{eva}} & =\kappa_{T}\left(\frac{D+\chi_{s}}{1-\chi_{s}}\right)\left(\frac{d^{2} f}{d \xi^{2}}\right)|\nabla \xi|^{2} \Delta b, \\
C(\epsilon) & =\left(1-\frac{d f}{d \xi}\right)\left(\frac{D+\chi_{s}}{1-\chi_{s}}\right)\left(q_{l}^{c}\right)^{-1} \nabla\left(\mathbf{j}_{\mu} \Delta b\right) .
\end{aligned}
$$

In the limit $\epsilon \rightarrow 0$, the term $(1-d f / d \xi)$ in Eq. (A3c) is nonzero only in regions without liquid water $(\ell=0)$, where the sedimentation flux is zero, and therefore the correction term vanishes in the whole domain, $C(\epsilon \rightarrow 0)=0$.

\section{REFERENCES}

Ackerman, A. S., M. P. Kirkpatrick, D. E. Stevens, and O. B. Toon, 2004: The impact of humidity above stratiform clouds on indirect aerosol climate forcing. Nature, 432, 1014-1017, doi:10.1038/nature03174.

_ - and Coauthors, 2009: Large-eddy simulations of a drizzling, stratocumulus-topped marine boundary layer. Mon. Wea. Rev., 137, 1083, doi:10.1175/2008MWR2582.1.

Albrecht, B. A., 1989: Aerosols, cloud microphysics, and fractional cloudiness. Science, 245, 1227-1230, doi:10.1126/ science.245.4923.1227.

Bretherton, C. S., 1987: A theory for nonprecipitating moist convection between 2 parallel plates. Part I: Thermodynamics and linear solutions. J. Atmos. Sci., 44, 1809-1827, doi:10.1175/ 1520-0469(1987)044<1809:ATFNMC>2.0.CO;2.

_ tation, entrainment efficiency, and subtropical stratocumulus albedo. Geophys. Res. Lett., 34, L03813, doi:10.1029/ 2006 GL027648.

Caldwell, P., and C. S. Bretherton, 2009: Large-eddy simulation of the diurnal cycle in southeast Pacific stratocumulus. J. Atmos. Sci., 66, 432-449, doi:10.1175/2008JAS2785.1.

Deardorff, J. W., 1970: Preliminary results from numerical integrations of the unstable planetary boundary layer. J. Atmos. Sci., 27, 1209-1211, doi:10.1175/1520-0469(1970)027<1209: PRFNIO $>2.0 . \mathrm{CO} ; 2$.

De Lozar, A., and J. P. Mellado, 2013: Direct numerical simulations of a smoke cloud-top mixing layer as a model for stratocumuli. J. Atmos. Sci., 70, 2356-2375, doi:10.1175/JAS-D-12-0333.1.

— and - 2014: Cloud droplets in a bulk formulation and its application to buoyancy reversal instability. Quart. J. Roy. Meteor. Soc., 140, 1493-1504, doi:10.1002/qj.2234.

_- and _- 2015a: Evaporative cooling amplification of the entrainment velocity in radiatively driven stratocumulus. Geophys. Res. Lett., 42, 7223-7229, doi:10.1002/ 2015 GL065529. 
, and - 2015b: Mixing driven by radiative and evaporative cooling at the stratocumulus top. J. Atmos. Sci., 72, 4681-4700, doi:10.1175/JAS-D-15-0087.1.

Geoffroy, O., J.-L. Brenguier, and F. Burnet, 2010: Parametric representation of the cloud droplet spectra for LES warm bulk microphysical schemes. Atmos. Chem. Phys., 10, 4835-4848, doi:10.5194/acp-10-4835-2010.

Gerber, H., G. Frick, S. P. Malinowski, H. Jonsson, D. Khelif, and S. K. Krueger, 2013: Entrainment rates and microphysics in POST stratocumulus. J. Geophys. Res. Atmos., 118, 12094 12 109, doi:10.1002/jgrd.50878.

Hill, A. A., S. Dobbie, and Y. Yin, 2008: The impact of aerosols on non-precipitating marine stratocumulus. I: Model description and prediction of the indirect effect. Quart. J. Roy. Meteor. Soc., 134, 1143-1154, doi:10.1002/qj.278.

— , G. Feingold, and H. Jiang, 2009: The influence of entrainment and mixing assumption on aerosol-cloud interactions in marine stratocumulus. J. Atmos. Sci., 66, 1450-1464, doi:10.1175/ 2008JAS2909.1.

Klingebiel, M., and Coauthors, 2015: Arctic low-level boundary layer clouds: In-situ measurements and simulations of monoand bimodal supercooled droplet size distributions at the top layer of liquid phase clouds. Atmos. Chem. Phys., 15, 617-631, doi:10.5194/acp-15-617-2015.

Larson, V. E., K. E. Kotenberg, and N. B. Wood, 2007: An analytic longwave radiation formula for liquid layer clouds. Mon. Wea. Rev., 135, 689-699, doi:10.1175/MWR3315.1.

Lilly, D. K., 1968: Models of cloud-topped mixed layers under a strong inversion. Quart. J. Roy. Meteor. Soc., 94, 292-309, doi:10.1002/qj.49709440106.

Lock, A., 1998: The parametrization of entrainment in cloudy boundary layers. Quart. J. Roy. Meteor. Soc., 124, 2729-2753, doi:10.1002/qj.49712455210.

Lu, M. L., and J. H. Seinfeld, 2005: Study of the aerosol indirect effect by large-eddy simulation of marine stratocumulus. J. Atmos. Sci., 62, 3909-3932, doi:10.1175/JAS3584.1.

Malinowski, S. P., M. Andrejczuk, W. W. Grabowski, P. Korczyk, T. A. Kowalewski, and P. K. Smolarkiewicz, 2008: Laboratory and modeling studies of cloud-clear air interfacial mixing: Anisotropy of small-scale turbulence due to evaporative cooling. New J. Phys., 10, 075020, doi:10.1088/1367-2630/10/7/ 075020 .

Mellado, J. P., 2010: The evaporatively driven cloud-top mixing layer. J. Fluid Mech., 660, 5-36, doi:10.1017/S0022112010002831.

- B. Stevens, H. Schmidt, and N. Peters, 2009: Buoyancy reversal in cloud-top mixing layers. Quart. J. Roy. Meteor. Soc., 135, 963-978, doi:10.1002/qj.417. and - 2010: Two-fluid formulation of the cloudtop mixing layer for direct numerical simulation. Theor. Comput. Fluid Dyn., 24, 511-536, doi:10.1007/s00162-010-0182-x.

Moeng, C., 2000: Entrainment rate, cloud fraction, and liquid water path of PBL stratocumulus clouds. J. Atmos. Sci., 57, 3627-3643, doi:10.1175/1520-0469(2000)057<3627:ERCFAL > 2.0.CO;2.

$\longrightarrow$, P. P. Sullivan, and B. Stevens, 1999: Including radiative effects in an entrainment rate formula for buoyancy-driven PBLs. J. Atmos. Sci., 56, 1031, doi:10.1175/1520-0469(1999)056<1031: IREIAE $>2.0 . \mathrm{CO} ; 2$

Petty, G. W., 2006: A First Course in Atmospheric Radiation. Sundog Publishing, $452 \mathrm{pp}$.

Rogers, R. R., and M. K. Yau, 1989: A Short Course in Cloud Physics. 3d ed. Elsevier, 304 pp.

Sandu, I., J.-L. Brenguier, O. Geoffroy, O. Thouron, and V. Masson, 2008: Aerosol impacts on the diurnal cycle of marine stratocumulus. J. Atmos. Sci., 65, 2705-2718, doi:10.1175/ 2008JAS2451.1.

Shao, Q., D. A. Randall, C. Moeng, and R. E. Dickinson, 1997: A method to determine the amounts of cloud-top radiative and evaporative cooling in a stratocumulus-topped boundary layer. Quart. J. Roy. Meteor. Soc., 123, 2187-2213, doi:10.1002/ qj.49712354403.

Siems, S. T., and C. S. Bretherton, 1992: A numerical investigation of cloud-top entrainment instability and related experiments. Quart. J. Roy. Meteor. Soc., 118, 787-818, doi:10.1002/ qj.49711850702.

Stevens, B., 2002: Entrainment in stratocumulus-topped mixed layers. Quart. J. Roy. Meteor. Soc., 128, 2663-2690, doi:10.1256/ qj.01.202.

_- and Coauthors, 2005: Evaluation of large-eddy simulations via observations of nocturnal marine stratocumulus. Mon. Wea. Rev., 133, 1443-1462, doi:10.1175/MWR2930.1.

Turton, J., and S. Nicholls, 1987: A study of the diurnal variation of stratocumulus using a multiple mixed layer model. Quart. J. Roy. Meteor. Soc., 113, 969-1009, doi:10.1002/qj.49711347712.

Twomey, S., 1974: Pollution and the planetary albedo. Atmos. Environ., 8, 1251-1256, doi:10.1016/0004-6981(74)90004-3.

van Zanten, M. C., and P. G. Duynkerke, 2002: Radiative and evaporative cooling in the entrainment zone of stratocumulusThe role of longwave radiative cooling above cloud top. Bound.Layer Meteor., 102, 253-280, doi:10.1023/A:1013129713315.

_ B. Stevens, G. Vali, and D. H. Lenschow, 2005: Observations of drizzle in nocturnal marine stratocumulus. J. Atmos. Sci., 62, 88-106, doi:10.1175/JAS-3355.1.

Wood, R., 2012: Stratocumulus clouds. Mon. Wea. Rev., 140, 2373 2423, doi:10.1175/MWR-D-11-00121.1. 